\title{
Singen macht glücklich und hält gesund
}

\section{Michaela Wurmehl}

Dr. med., Fachärztin für Innere Medizin, FMH

Unter dem Motto «Singen macht glücklich und hält gesund» (Prof. Dr. Gunter Kreutz) möchten sich die Ärztinnen und Ärzte des Deutschen Ärztechores und des Deutschen Ärzteorchesters nicht immer nur mit Krankheiten befassen, sondern auch einmal auf eine ganz andere Art Gesundheitsprophylaxe betreiben: Sie möchten die Aus- und Weiterbildung musikalisch begabter junger Menschen in ihrer Begeisterung zu singen und zu musizieren durch den Ertrag eines Benefizkonzertes unterstützen. So sollen mit dem Erlös des Benefizkonzertes die beiden in Basel heimischen Vereine Knabenkantorei Basel und Juventus Musica Basel finanziell unterstützt werden.

Die Mitglieder von Chor und Orchester sind Ärzte, Studierende und Angehörige medizinverwandter Berufe aus Deutschland und der Schweiz.

Die schon sehr lange gepflegte Tradition des Singens und der Stimmbildung in der Knabenkantorei Basel, deren Resultat jeweils in berührenden Konzerten bewundert werden kann, hat schon zahlreichen Jungsängern sehr grosse Freude bereitet und ihr Leben und ihre Gesundheit entscheidend positiv beeinflusst. Die Knabenkantorei Basel ist aus den 1927 gegründeten Singknaben der evangelisch-reformierten Kirche BaselStadt hervorgegangen. Der Chor besteht aus rund 45 Knaben- sowie 35 Männerstimmen. Die Männerstimmen sind in der Regel ehemalige Knabenstimmen, die auf eine langjährige Chorerfahrung zurückblicken. Die Sänger setzen einen wesentlichen Teil ihrer Freizeit für die Musik ein: Neben den regelmässigen Proben zweimal wöchentlich kommen Gottesdienste, Konzerte, Konzertreisen, Radio- und TV-Aufnahmen sowie CD-Einspielungen dazu. Viele erlernen ausserdem ein Musikinstrument. Zur Vertiefung des Chorrepertoires finden jährlich ein Chorlager sowie ein bis zwei Wochenendproben pro Jahr statt. Der Chor ist konfessionell neutral, gesungen werden geistliche und weltliche Werke.

Aus dieser Tradition der Knabenkantorei heraus ist durch die Initiative des KKB-Sängers Raphael Ilg Juventus Musica entstanden, nachdem er 2010 mit 60 Freun- den und Schulkollegen des Gymnasiums Münchenstein als Maturarbeit den 1. Teil des Elias von Felix Mendelssohn einstudiert und aufgeführt hat. Die Konzerte waren so erfolgreich, dass im Jahr darauf ein weiteres Projekt folgte: der Lobgesang, ebenfalls von Mendelssohn. Danach war die Idee geboren, einen offiziellen Verein zu gründen. Seither musizieren begabte Jugendliche der Region Basel im Chor und Orchester von Juventus Musica mit Begeisterung in ihrer Freizeit weiterhin gemeinsam unter der Leitung von Raphael Ilg, der zurzeit an der Musikhochschule Basel Oboe und Dirigieren studiert, und bringen grosse Werke der klassischen Musikliteratur mit ebenfalls jugendlichen Solisten zur Aufführung: 2013 das Requiem von A. Dvořák und 2014 sowohl den 95. Psalm von F. Mendelssohn als auch das eher unbekannte Miserere von E.T.A. Hoffmann (CD-Aufnahme).

2015 stehen neben zwei Orchesterkonzerten mit einer Uraufführung im Juli auch eine Reise nach Lissabon, auf Einladung des Lisbon Youth Music Festival, sowie ein gemeinsames Weihnachtskonzert mit der Münsterkantorei Basel auf dem Programm. Einzelne Mitwirkende studieren Musik, die meisten Jugendlichen sind jedoch sehr gute Amateurmusiker. Sämtliche organisatorischen Angelegenheiten werden von den Jugendlichen selbst übernommen.

\section{Mit dem Erlös des Benefizkonzertes werden} die Knabenkantorei Basel und Juventus Musica Basel unterstützt.

Ganz besonders freuen sich die Ärztinnen und Ärzte des Deutschen Ärztechores und des Deutschen Ärzteorchesters, dass ihr Konzert durch Sänger dieser beiden Ensembles, nämlich 3 Knabensoli und Männerstimmen sowie Alumni der Knabenkantorei Basel wie auch von Juventus Musica, aktiv singend unterstützt wird und so der Aufführung ein ganz besonderer Klang und Glanz verliehen wird.

Der Deutsche Ärztechor hat seine Wurzeln im Deutschen Ärzteorchester und wurde aus dem Wunsch heraus gegründet, auch Oratorien und grosse Werke für Chor und Orchester mit Ärzten aufzuführen. Zum ers- 


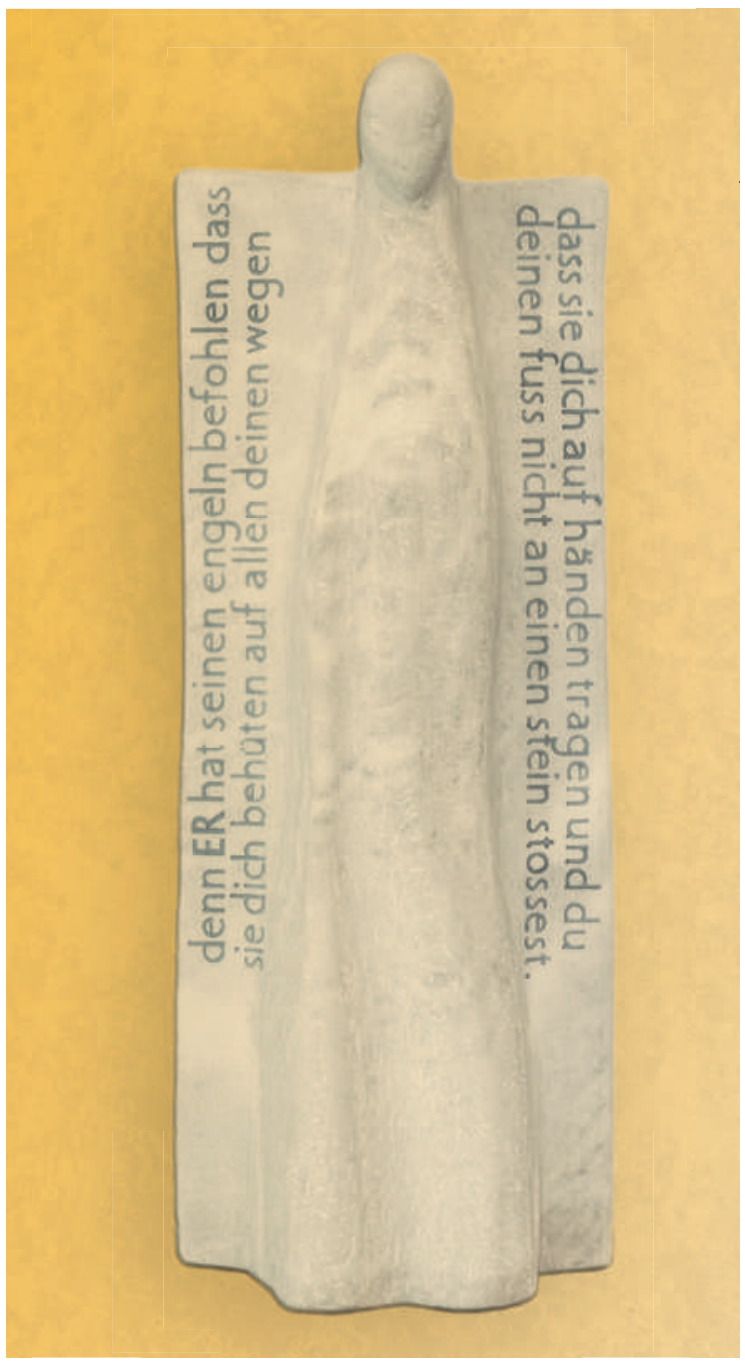

Das Oratorium Elias von Felix Mendelssohn Bartholdy beruht auf Worten des Alten Testaments.

ten Mal traf sich der Chor mit dem Deutschen Ärzteorchester im Dezember 2007 auf der Nordseeinsel Amrum, um gemeinsam das Weihnachtsoratorium von J.S. Bach einzustudieren und aufzuführen. Der grosse Erfolg ermutigte zur Gründung des Chores als Verein und zu weiteren gemeinsamen Konzerten der beiden Ensembles in verschiedenen deutschen Städten. Mit dem Bayerischen Ärzteorchester wurde 2011 das Requiem von G. Verdi in München, Bamberg, Bayreuth und Landau aufgeführt. Seine erste Auslandstournee mit dem Deutschen Requiem von J. Brahms führte den Deutschen Ärztechor 2012 in die norditalienischen Städte Lomello, Venedig, Parma, Mantua und Mailand. Weitere Konzerte mit Begleitung durch verschiedene Ärzteorchester waren: F. Mendelssohn: Lauda Sion / A. Dvořák: Stabat mater, J.S. Bach: Johannespassion,
J. Haydn: Die Schöpfung, G.F. Händel: Der Messias, Ch. Gounod: Cäcilienmesse / H. Berlioz: Te Deum, W.A. Mozart: Requiem und ein A-capella-Konzert mit verschiedensten Liebesliedern. Die inzwischen über 300 Mitglieder des Chores sind sangesbegeisterte und musikalisch hochbegabte Ärzte und Studierende der Medizin sowie Angehörige medizinverwandter Berufe aus allen Teilen Deutschlands und der Schweiz.

Das Deutsche Ärzteorchester wurde im Jahr 1989 in München gegründet. Es hat in den letzten Jahren in vielen Städten Deutschlands konzertiert, u.a. in München (Gasteig und Herkulessaal), Hamburg (Laeiszhalle), Stuttgart (Liederhalle) und Braunschweig (Staatstheater) u.a. Die Besetzung entspricht einem vollen Sinfonieorchester. Im Augenblick gehören ihm ca. 150 Mitglieder an - überwiegend Ärztinnen und Ärzte aller Fachrichtungen, Angehörige von medizinassoziierten Berufen und Medizinstudenten, die sich drei- bis viermal jährlich projektbezogen zu Probenphasen mit anschliessendem Konzert treffen. Daneben finden zahlreiche kammermusikalische Aktivitäten statt. Viele der Orchestermitglieder verfügen zusätzlich zur medizinischen über eine professionelle musikalische Ausbildung. Das Deutsche Ärzteorchester finanziert sich aus Mitglieds- und Seminarbeiträgen sowie über Sponsoren und Förderer.

Für beide Ensembles ist es Brauch, für einen guten Zweck zu musizieren. So verbinden sie ihre Freude an der Musik mit der ärztlichen Grundhaltung, Gesundheitsprophylaxe zu betreiben und Menschen in sozialer oder krankheitsbedingter Schwierigkeit oder Not zu unterstützen.

\section{Benefizkonzert}

Samstag, 31. Oktober 2015, 19.30 Uhr, Stadtcasino Basel Felix Mendelssohn Bartholdy

ELIAS. Oratorium nach Worten des Alten Testaments, Opus 70

Miriam Meyer - Sopran

Judith Thielsen - Alt

Michael Connaire - Tenor

Konstantin Heintel - Bass (Titelpartie)

Deutscher Ärztechor, Choreinstudierung Uta Singer

Knabensoli und Männerstimmen der Knabenkantorei Basel und Juventus Musica Basel,

Deutsches Ärzteorchester, Gesamtleitung: Alexander Mottok

Vorverkauf: Bider und Tanner, ticket[at]biderundtanner.ch Weitere Informationen zu den musikalischen Vereinen unter www.knabenkantorei.ch; www.juventus-musica.ch;

www.aerztechor.de; www.aerzteorchester.de 